\title{
Collaborative Information Systems and Business Process Design Using Simulation
}

\author{
Ray J. Paul, Ray.Paul@Brunel.ac.uk \\ Alan Serrano, Alan.Edwin.Serrano-Rico@Brunel.ac.uk \\ Department of Information Systems and Computing, Brunel University, UK
}

\begin{abstract}
The Information Systems (IS) community promotes the idea that IS analyst should have a clear understanding of the way the organization operates before attempting to propose an IS solution. It is argued that to take a complete advantage of the underlying Information Technology (IT), organizations should first identify any process flaw and then propose a suitable IT solution. Similarly, many process design approaches claim that Business Process (BP) design should be done considering the advantages provided and the limitations imposed by the underlying (IT). Despite this fact research in these domains provides little indication of which mechanisms or tools can help $B P$ and IS analyst to understand the complex relationships amongst these two areas. This paper describes the insights gained during a UK funded research project, namely ASSESS-IT, that aimed to depict the dynamic relationships between IT and BP using simulation. One of the major limitations of the ASSESS-IT project is that it looked at relationship between BP and IT as a three layered structure, namely BP, IS and Computer Networks (CN), and did not explore in detail the relationships between $B P$ and IS alone. This paper uses the outcomes derived from this project and suggests that, is some cases, the relationship between BP and IT could be analyzed by looking at the relationship between BP and IS alone. It then proposes an alternative simulation framework, namely BPISS, that provides the guideline to develop simulation models that portray BP and IS behavior performance measurements, offering in this way an alternative mechanism that can help BP and IS analyst to understand in more detail the dynamic interactions between BP and IS domains.
\end{abstract}

Keywords: IS design, BP design, Simulation

\section{Introduction}

Business Processes (BP) became the focus of continuous improvement efforts during the 1940's [1].
Despite BP was studied throughout the years under various different disciplines it was not until the beginning of the 1990's when the process movement became stronger. Business Process Reengineering also named Process Redesign, or Process Innovation is today one of the most popular concepts in business management [2,3]. The study of business processes, however, is not isolated and has always been related to Information Technology (IT). IT is considered one of the most important enablers of process change. For example, in one of the first articles about BPR, Davenport and Short [4] argue that together, processes and information technology can be seen as a new industrial engineering that may revolutionize the way in which organizations operate.

Most of the advocators of the business process reengineering movement highlight the importance of the role that IT plays in the reengineering process. Many argue that IT should be seen as an enabler of organizational change rather than as a tool to implement business processes [2]. Childe et al. [5] for example, state that the initiative to move towards BPR in many cases originates from the IT departments. In one of the first empirical studies on IT-enabled BPR [6] it is claimed claim that the success of IT to enable BPR lies in Information Systems (IS) strategy integration. They contend that the success of ITenabled BPR efforts will succeed only if they are directed through a strong integration with strategy. This relationship, however, is not fully explored in most of the existing IS methodologies, or in the BP domain. For example, Davenport's BPR methodology [2] identifies different ways that IT can be used to improve process performance. Davenport's framework, however, does not supply information that indicates how to assess the impact that the insertion of a new IS may have on the processes or which techniques can be used to investigate this relationship. Similarly, Kettinger et al. [7] proposed the Stage-Activity (S-A) framework for BPR. This framework, though, focuses almost entirely on identifying techniques and tools that can be used in each of the stages for the design of BP and barely mentions how to assess the impact that the 
use of IS may have on the processes or how to achieve the coordination between process and information techniques.

Trying to overcome these problems, practitioners in these domains have proposed different approaches. For example, Painter et al. [8] proposes a simulationbased methodology, namely BPR-II, to assess the impact of changes to business processes and the IT infrastructure that support those processes. They argue that the BPR-II methodology and an accompanying automated support environment will provide the ability to link models of both business process and the supporting IT infrastructure. Painter et al. propose a three-layered structure that defines the relationship between BP and IT: BP, computer networks (CN), and IS. To this end, it is proposed the use of the IDEF3 method as the key mechanism for process capture. IDEF3 process descriptions are used in this methodology to capture a definition of the process at all three levels, namely business process, application (IS) and network processing, and to directly generate the structure and logic of simulation models reflecting these levels. A major limitation of this approach is that it requires the development of three models as well as the refinement of the simulation models derived from such models.

This research assumes that in order to design better business process (BP) and information technology (IT) solutions, practitioners in these domains should coordinate the design of BP and IT. To this end, it is considered that the relationship between organizational processes and the underlying information technology could be seen as a three layered structure (see Figure 1).

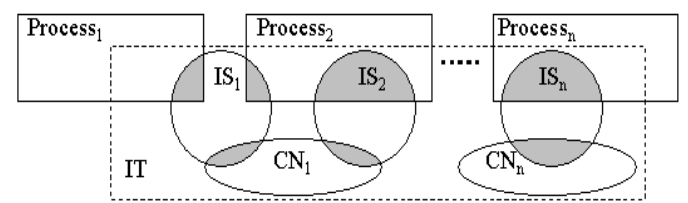

Figure 1. BP, IS and CN Relationships

Business processes represents the first layer. The second layer is formed by the organization's information systems (IS) that support many of the activities involved in the business processes. Finally, the last layer is the computer network (CN) infrastructure that is used to exchange the information generated and handled by IS. A problem related to these relationships is that changes on any of these three layers could have repercussions on the others and that current modeling techniques in these domains were not able to predict these effects.
To address this problem, this paper uses the insights gained from a UK funded research project, namely ASSESS-IT, that used Business Process Simulation and Computer Network Simulation techniques to model the dynamic behavior of the relationships between BP, IS and CN. This paper takes the findings from the project and uses the knowledge gained to propose a new framework that applies business process simulation techniques to depict the interactions between business processes and information systems, namely the BPISS framework.

The case study used to test the framework is not designed to be exhaustive but rather to show that the development of simulation models to capture the behavior of business process and information systems is possible, and that the results provide relevant information to be used for IS and BP design. The case study also provides opportunity to discuss the advantages and limitations of the proposed frameworks.

\section{Modeling process and information technology using discrete-event simulation}

The fact that simulation has been successfully used in the process domain posits this technique as one possible candidate to address the business process and information technology integration challenge. The BP domain has largely used BPS models to assess the impact that changes to business processes may have on the organisation and to explore different BP scenarios [9,10,11,12,13]. A Business Process Simulation (BPS) exercise, though, do not consider how new IT may affect business process performance. The ASSESS-IT project investigated the suitability of using discrete event simulation techniques in a modelling framework to depict BP and IT interactions. It investigated the possible ways to link CNS models results to a BPS model so that the impact that IT may have on BP was reflected on the BPS model. Current CNS are able to model both the computer network behaviour and they way IS applications may affect network behaviour [14]. Therefore, the modelling framework proposed in the ASSESS-IT project combines the ability of BPS models to portray the organisational aspects and relates this model to a CNS model to address both IS and CN factors that may have an impact on BP process performance. The following section explains the steps propose in the ASSESS-IT framework. 


\section{The ASSESS-IT framework}

The ASSESS-IT project assumed that a problem related to the relationships between business process, information systems and computer networks is that changes on any of these three layers would have repercussions on the others and that current modeling techniques in these domains were not able to predict these effects. To address this problem, ASSESS-IT proposed a simulation framework to depict the impact that changes to the business processes may have on the information systems and computer network layers and vice versa. The ASSESS-IT framework recommended a series of steps to develop simulation models that exchange performance measurements of $\mathrm{BP}$ and $\mathrm{CN}$ simulation models, reflecting in this way the impact that changes to IT infrastructure may have on BP performance.

The ASSESS-IT framework is based on Banks et al. [15] simulation steps. The simulation steps proposed in Banks et al., thought, are designed to develop a single simulation model. Because the ASSESS-IT framework aimed to develop two simulation models and to share the results obtained from each other so that changes in one model are reflected in the other, the following modifications to the aforementioned steps were derived (see Figure 2).

- The ASSESS-IT framework duplicates Banks et al's steps for each of the models. The left column in Figure 2 depicts the steps to follow for the BP model, whilst the right column depicts the steps to follow for the IT model.

- To coordinate the design of BP and IT so the corresponding models include both BP and IT views it was proposed that the problem formulation and setting of objectives and overall project plan steps should be performed together for both business process and computer network models. In this way both BP and IT analysis can share common information that can be used for both BP and IT models.

- To coordinate the conceptualisation of the BP with the $\mathrm{CN}$ models and vice versa so they reflect both process and information technology issues a new step, BP/IT model conceptualisation (4a and grey coloured in Figure 2) is introduced. This step aims to verify that IT issues are considered in BP design and vice versa.

- $\quad$ For the BP model, steps 5, 6, and 7 are performed as suggested in Banks et al.. As for the CN model, all subsequent steps follow the framework suggested in Banks' framework.

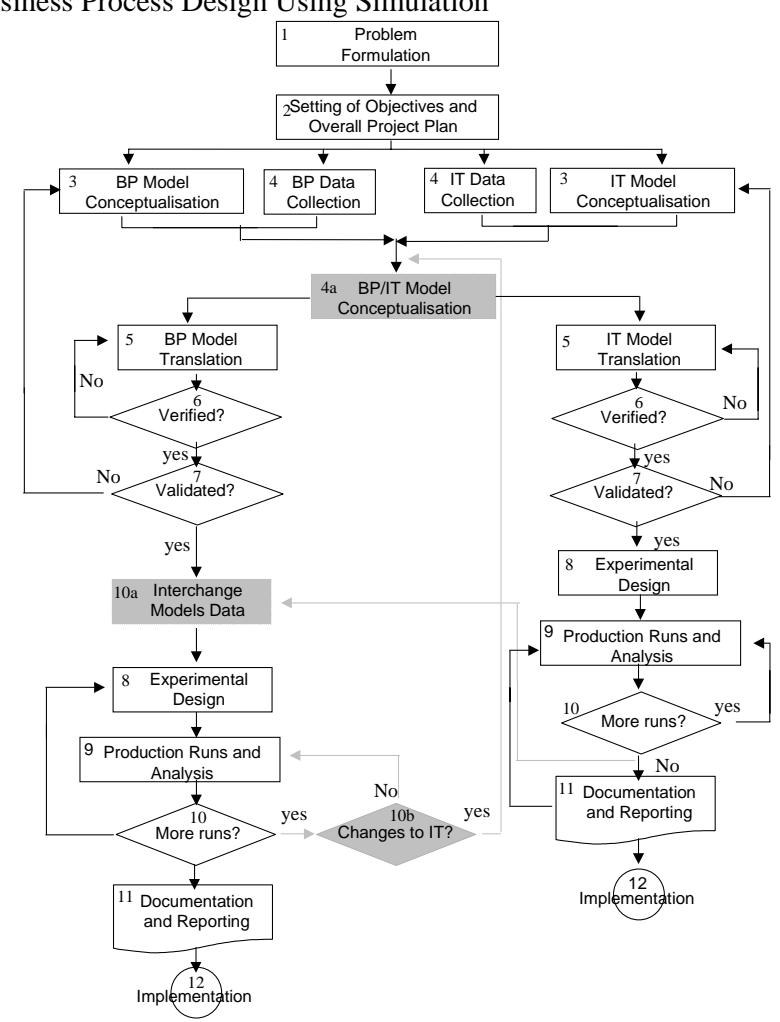

Figure 2. ASSESS-IT Simulation Framework

- A new step (10a and grey coloured in Figure 2), interchange data between models, is introduced in the BP model steps. Before undertaking the experimental design step, the BP modeller should wait for the input from the $\mathrm{CN}$ model results (e.g. transmission times over specific network conditions). This information needs to be considered for the experimentation design phase in the business process model.

- If more runs are needed in step 10 in the business process model, another decision needs to be made (step 10b and grey shadowed in Figure 2). If the decision in the business process implies changes to the IT, both models need to start at the step 4b, otherwise the BP steps continue normally. It is expected, though, that changes to the models will not be of great significance. Therefore, the following steps should be completed quicker than in the first round.

\section{The ASSESS-IT project case study}

The case study used to test the ASSESS-IT framework consists of two collaborating organizations in Greece. One company is a branch of a major multinational pharmaceuticals organization (we will 
refer to this company as Org-A), while the other is a small-sized regional distributor of Org-A's products (we will refer to this company as 'Org-B').

The case study was carried out within a single business unit, which deals with hospital consumables. The business unit does not produce products but imports them from other Org-A production sites across Europe. The goods are stored in a warehouse that operates as a central dispatch point for all products, which are then distributed to the company's customers via a network of collaborating distributors. One of these distributors is Org-B.

Org-B is a small company that has signed an agreement to act as Org-A's exclusive distributor of Medical unit products. The agreement states that OrgB's responsibilities include:

1. Receiving orders from Org-A customers.

2. Maintaining an adequate inventory of products that fulfill the orders.

3. Distributing the ordered products to customer premises.

Due to the nature of the products, Org- $\mathrm{B}$, as the company in charge of delivering products, has to operate within rigorous deadlines. The agreement between the companies, stipulates that each order has to be fulfilled within 24 hours for products delivered within the city of Thessaloniki, or within 48 hours for the rest of northern Greece.

Org-A management has noted, however, that these targets are rarely met in practice. A brief analysis by the companies seemed to attribute the problems to some inefficiencies within the ordering system as well as difficulties being experienced by Org-B in maintaining their inventory at an optimal level. In addition to this the communication system between the two companies was also seen as slow and cumbersome. The effects that these inefficiencies caused were seen as a major source of customer dissatisfaction, so an indepth analysis of the problem was commissioned. The main objectives of the ASSESS-IT study were:

1. To examine the existing business processes that were felt to be responsible for long lead times for order fulfillment.

2. To determine the sources of problems and propose alternative solutions.

3. To evaluate the potential of introducing appropriate IT to improve communication between the two companies.

In order to calculate the business effects of changing the underlying IT, the ASSESS-IT framework aimed to develop a computer network model, including IS design, identify the information that may be relevant to the BPS model and incorporate it in the latter. The framework is divided into two major phases. The first phase develops the Computer
Network Simulation model. The second phase develops the Business Process Simulation Model and uses the outputs of the CNS model to reflect the impact of the IT in the BP. A complete description of the ASSESS-IT framework and analysis of the results can be found in [16].

\section{ASSESS-IT: Lessons learned}

The results obtained from ASSESS-IT showed that the impact that the computer network infrastructure were heavily dependent on time. These results also demonstrated that due to current network technologies, the information systems that could suffer from changes to network architecture are those that depend on time. The type of systems that the ASSESS-IT approach aimed to address, however, do not fit within this category. The experiments showed that changes to the network infrastructure and to other parameters (network traffic) did not have a considerable effect on IS performance, and consequently, did not affect the BP performance. Similar results were found in the business process-modeling phase. The results obtained in this phase showed that using IT to improve business response time does not necessarily improve process performance. The experiments did not show a significant improvement on business process performance despite the fact that the time for those activities that used the new IS was dramatically reduced. A deeper analysis of this situation suggested that the problem was due to the fact the ASSESS-IT approach concentrated on depicting the way IT affects processing time, but not in the way IT affects process performance. Business process modeling experiments showed that time was not a parameter that could affect process performance [17]. The experiments showed, however, that there are other IS parameters that affect BP performance. For example, once it was detected that the backordering process was a major system bottleneck, it was proposed to use the EDI system to alleviate this problem. Therefore, the IS was designed, amongst other things, to reduce the number of backorders. This information, though, could not be reflected in the business process model because the BP model was designed to represent the percentage of backorders as a fixed number and not dynamically. Reducing the backorders percentage manually (from $30 \%$ to $5 \%$ ) demonstrated that the reduction of backorders would reduce the overall processing time. This figure was directly related to the way the IS would handle the backordering process.

Two conclusions can be obtained from the ASSESSIT approach exercise. A) The computer network infrastructure does not affect the performance 
of information systems used to support organizational processes. Consequently, the overall business processes performance is not affected, in a significant way, by changes to the $\mathrm{CN}$ infrastructure. Therefore, the use of a computer network model is, in the context of the ASSESS-IT approach, unnecessary. B)The experimentation with different BP scenarios provided evidence that suggests that in order to portray the benefits that the use of an IS may bring to the business processes, it is necessary to obtain measurements of the way the IS behaves over time.

In order to provide a modeling approach that depicts the relationships and interactions between BP and IT, it is necessary to focus on the relationship between BP and IS alone. Furthermore, the insights gained from the experiments with different BP scenarios imply that the parameters that govern the relationship between business processes and information technology are not those that are related to time constraints but are instead those that are related to IS performance measurements. It was observed that time reduction on certain activities of the ordering process did not improve business process performance. On the other hand, IS performance measurements, such as the reduction of the number of backorders produced by the IS, improved the overall process performance. These facts lead us to think that a new BP/IT integrated approach was needed. Therefore, the new approach should focus on the relationship between BP and IS, and more importantly, should depict IS behavior measurements. This means, it is necessary to model IS performance. IS performance measurements are also known as information system's Non-Functional Requirements (NFR) [18,19]. Most IS modeling techniques, however, aim to depict functional requirements. Non-Functional Requirements, on the other hand, are not easy to represent in a measurable way, thus, a limited number of techniques and approaches can be found [20]. The results rendered by the ASSESS-IT approach highlighted the need to portray the dynamic behavior of the IS as it evolves over time. The following section presents the rationale for a new hypothesis that uses the ASSESS-IT framework results as the base to propose a new approach, namely BPISS Framework, that can be used to identify NFR that affect IS performance and to model the behavior of the IS and the BP as they evolve over time.

\section{The BPISS framework}

This paper uses the experience gained from the ASSESS-IT project, and proposes an alternative approach to address the limitations found. The new framework, namely BPISS (Business Process and Information Systems Simulation), attempts to portray the behavior of both IS and BP using discrete-event simulation techniques. The major objective of the BPISS is to provide guidelines to develop a simulation model that provides stochastic measurements of the way business process and information system behave, thus assessing the impact that IS may have on BP. To achieve this objective, the BPISS framework is divided into sixteen tiers (see Figure 3).

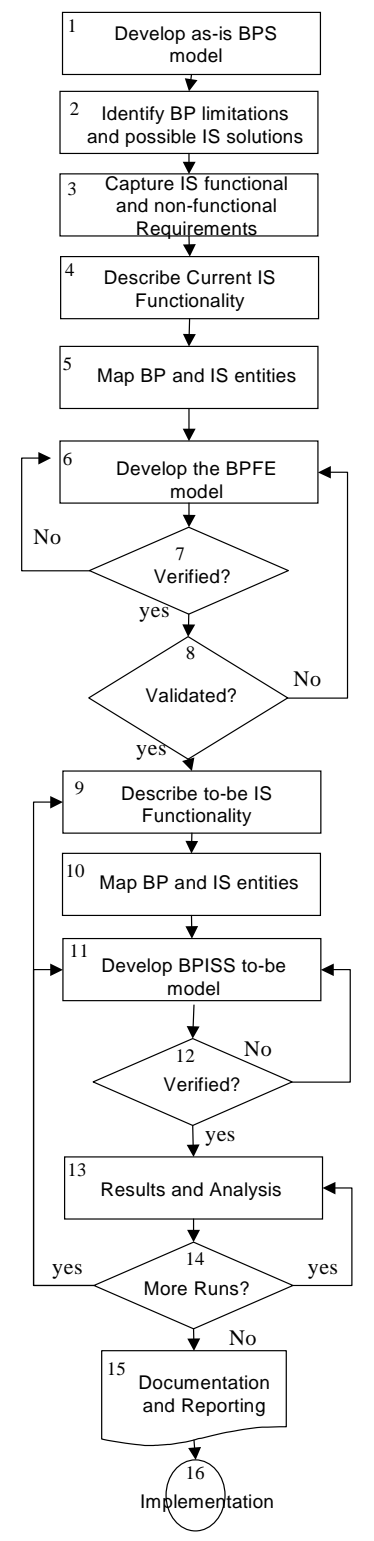

Figure 3. The BPISS Framework

The first tier can be considered as a simulation study on its own where the major aim is to develop a 
model of the way current business processes operate (as-is BP model). The results from ASSESS-IT showed that the development of as-is BPS models could help to identify possible process limitations and areas of improvement, including possible IT solutions. The BPS model derived from this tier will be used in subsequent tiers to a) identify process limitations, b) propose alternative BP and IT solutions, c) identify non-functional requirements, and d) validate the BPISS model. Tiers Two to Eight are the guidelines to develop an as-is BPISS model. The BPISS model considers the way the functionality of the IS supports business processes. Because this is an as-is model, the functionality of the current system should be portrayed. If the business processes are not supported by IT, the functionality of the manual system should be modeled instead. Tiers Nine to Twelve are the guidelines to develop the to-be BPISS model considering the functionality of the proposed IS solution together with the corresponding changes to the BP. Tiers Thirteen to Sixteen use Banks et al [15] simulation steps (steps nine to twelve) to conclude the simulation study.

Because the major aim of this paper is to understand the dynamic interactions between processes and information systems, the following subsections are a detailed description of how tiers Two to Eight of the BPISS framework aim to achieve this aim. A full description of the BPISS framework tiers can be found in [16].

\subsection{Tier Two: Identifying BP limitations and possible IS solutions}

Aim. The as-is BPS model developed in Tier One will be used in this tier to identify current process limitations and to identify those processes where performance can be improved by the insertion of IS. In cases where the organization is already supported by an IS, this step will identify those processes where IS are currently used.

Activities and Techniques. To identify process limitations the performance measurements obtained from the BPS model should be assessed against organizational performance goals the modeler can identify those processes where performance measurements are not satisfactory. Experimentation, with different scenarios can be used to identify possible causes that produce low process performance, such as poor process design, inappropriate use of resources, and so on. Once the proposed scenarios are tested, and process-related problems are identified, the insertion of IS should be considered next. To do so, IS and BP analysts should coordinate efforts so the functionality of the new IS addresses the limitations found in Tier One. Therefore, this tier also aims to provide a general description of what the proposed IS system is expected to do, in other words the functionality of the IS. In doing so, BP and IS modelers will be able to assess which processes can be benefited from the insertion of the IS and the ways it may improve process performance.

\subsection{Tier Three: Identify and capture IS non- functional requirements}

Aim. This tier aims to identify and capture the expected performance of the information system. This tier aims to use the BPS model to identify other performance measurements that may have been overlooked in Tier Two.

Activities and Techniques. The BPISS framework will use the BPS model develop in Tier One to identify performance requirements. To achieve this aim, BP and IS modelers should analyze those processes and activities that were considered to be affected by the proposed IS, previously identified in Tier Two. A closer analysis of the behavior of such processes and activities can provide useful information to identify other IS performance requirements that could have been overlooked using current IS modeling techniques. The IS functionality models developed in Tier Two should be complemented with the findings of this tier.

\subsection{Tier Four: Describe current IS functionality}

Aim. This tier aims to capture three major aspects of the current IS. One, to identify the overall workflow of the IS activities that are related to BP so this can be simulated in the as-is BPISS model. Two, to identify and understand how the data manipulated by the IS may affect BP entities, thus BP performance. Three, to identify the IS operations performed in during the process flow, so they can be represented in the BPISS model.

Activities and Techniques. This tier advocates the use of three UML modeling techniques: Activity models to capture the system's flow and identify relationships between activities. Collaboration diagrams to identify the collaboration between IS components and the IS operations performed. Finally, class models will be used to identify those classes that are related to the business entities. Although the BPISS framework advocates the use of UML, other IS modeling techniques that provide same information can also be used. 


\subsection{Tier Five: Map BP and IS entities}

The ASSESS-IT framework defined two different types of entities. Record Entities (RE) which are those entities found at the business level. RE usually represent objects that contain information and are used in the BP model to represent process behavior. The second entity type defined in the ASSESS-IT framework are Field Entities (FE). FE are the collection of entities that represent the information contained in a RE.

Aim. This tier aims to:

1. Identify all RE used in the business process level and map them to their corresponding FE.

2. Map the FE to the corresponding process/activity in the BP model.

3. Identify the operations performed during these activities and that affect RE or FE.

Activities and Techniques. In Tier Four of the BPISS framework a number of IS models that depict IS entities were produced. This tier will use those models to create a table, namely Record Entity/Field Entity Relationship (REFER), which relates the entities that are used in the BP model, their corresponding FE, the activities where FE are used to control the IS flow, and the operations performed when using these FE. The first step to build the REFER table is to identify RE and their corresponding FE. The activity diagrams developed in Tier Four will be used to identify which processes and/or activities use the FE identified before. The activities/processes are registered in the process/activity column. Finally, collaboration diagrams will be used to identify the IS operations that use or transform the FE in each of the activities. In doing so, each row of the REFER table provides information about a given FE, to which RE it is linked, in which activities it is involved, and which is the aim pursued by the IS when using the FE (IS operation). An example of a REFER table is shown below.

Table 1. A REFER Table Example

\begin{tabular}{|c|c|c|c|}
\hline RecordEntity & FeldEntity & Process/Activity & Operations \\
\hline Order & product id & check warehouse activity & Toidentify the product requested \\
\hline & product_quantity & check warehouse activity & $\begin{array}{l}\text { Todheck product_stock stock levels } \\
\text { against product stock }\end{array}$ \\
\hline & Product_stock & check werehouse activity & $\begin{array}{l}\text { Tocheck product_stockstock levels } \\
\text { against product stock }\end{array}$ \\
\hline
\end{tabular}

\subsection{Tier Six: Develop the as-is BPISS model}

Aim. This tier aims to develop the as-is BPISS model using the information collected in previous tiers, in particular, the information captured in the REFER table. To develop the as-is BPISS model the modeler needs to identify the RE and FE that are related to business performance and the business rules, including informational aspects, that may affect them.

Activities and Techniques. The BPISS framework considers the IS as a black box (see Figure 4). The relationship between $\mathrm{BP}$ and IS can be seen as relationships between input and output interfaces in each process and/or activity with the corresponding interfaces in the IS. For instance, the relationship between BP output-IS input interfaces is used to obtain information contained in BP entities (FEs) to perform the corresponding operations in the IS. Subsequently, the relationship between IS output-BP input interfaces is used to reflect the changes that the IS produces on the BP entities. It can be observed in Figure 4 that any input to the IS is reflected at any activity within all processes.

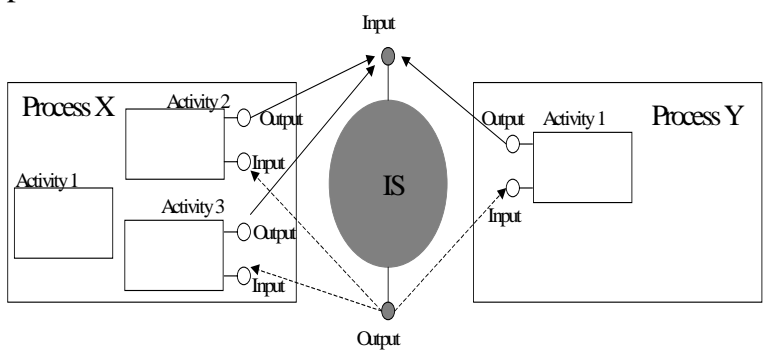

Figure 4. BP and IS Relationship in the BPISS Framework

To develop the as-is BPISS model, this tier will modify the as-is BPS model developed in Tier One so it includes the information system behavior. In doing so, it is assured that the components used in the as-is BPS model, such as data distributions, resources, and entities are consistent in both models. The development of the as-is BPISS model is divided in the following steps:

One. Represent FE in the RE. FE should be represented in the BPISS model as attributes of RE. This information can be obtained from the REFER table.

Two. Program the functionality of the system. The modeler needs to insert programming code that represent the business conditions that produce changes to $\mathrm{FE}$ and $\mathrm{RE}$ together with the consequences of these changes. Most of current BPS software provides the facility to add programming code to any activity and make changes to different model elements, including entities and attributes. Furthermore, the modeler can chose the simulation conditions when the code should be processed. For example, the code can be processed when an activity accepts an entity or when the latter is released from the activity. This adds the flexibility of choice to select the most suitable condition to run the code. To achieve this aim the modeler can use the information provided by the REFER tables, which 
indicate in which processes/activities the code should be inserted. In case that the information described in the REFER tables cannot provide enough details about the functionality of the system in a given activity, this information can be obtained analyzing the activity and collaboration diagrams developed in Tier Four.

\subsection{Tier Seven: Verify the as-is BPISS model}

Aim. This tier aims to ensure that the computerized representation of the model is correct.

Activities and Techniques. The BPISS model is, per se, a simulation model. Thus, existing techniques to verify simulation models apply to the BPISS model. Detailed information about verification techniques can be found in Banks et al. [15] and Law and Kelton [21].

\subsection{Tier Eight: validate the as-is BPISS model}

Aim. This tier aims to ensure that the model is an accurate representation of the real system.

Activities and Techniques. Because the as-is BPISS model is based on the as-is BPS model developed in Tier One, and the latter has already been validated, this tier aims to validate the as-is BPISS model against the as-is BPS model. To validate the asis BPISS model, performance measurements from the as-is BPISS model are compared against those from the as-is BPS model. Amendments to the BPISS model should be done until the results of the as-is BPISS model match, in the best possible way, the results from the as-is BPS model.

\section{Experimentation and results}

According to the ASSESS-IT notes the proposed IS should accomplish the aims described in the following list.

- The IS should automatically update inventory levels so a real-time inventory level could be monitored by both organizations.

- $\quad$ The IS should automatically produce a backorder whenever a given product is out of stock.

- When a backorder is produced or the inventory level of a given product is below the figures established by the organizations, the IS should request the replenishment of this product.

- When a replenishment cargo is delivered, the inventory levels should be updated and reflected in real-time.

Two IS non-functional requirements related with performance measurements were identified for the proposed IS. The first and most important requirement was requested by Org-A and establishes that the overall delivery time, including backorders, should be 24 hours, for products delivered within the city of Thessaloniki, or within 48 hours for the rest of northern Greece. This means that it is expected that the introduction of the new IS would reduce current delivery times so they fit the requirements previously mentioned. The second requirement was obtained during experimentation of the BPS model. It was detected that the backordering process was a major system bottleneck, and that delivery times depended on this process. It was demonstrated that when reducing the backorders percentage from $30 \%$ to $5 \%$, the overall processing time was significantly reduced. Therefore, a performance requirement that was not identified before is related to the percentage of backorders produced by the IS.

The results of the BPISS model reported a significant reduction in the totality of lead times, in particular, backorder lead times. Table 2 shows that backorder lead-time for both, Thessaloniki and Northern Greece were reduced in more than $80 \%$.

Table 2 BPISS Model Results

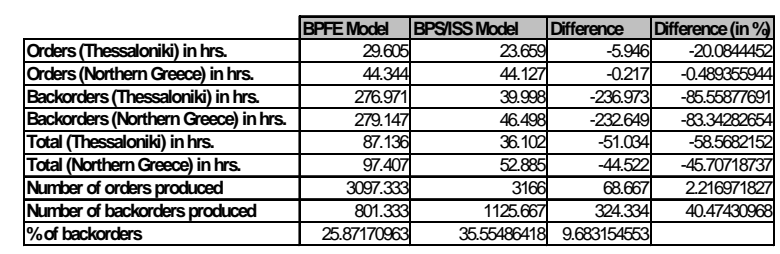

The reductions on lead-time, however, were still below the organizational targets. An interesting observation in Table 2 is that the percentage of backorders produced by the BPISS model reported an increase of nearly $10 \%$, a situation that contradicts the assumptions made in Step Two. A possible reason that causes this situation is that the minimum product stock level used in the model (10 products) produces a greater number of backorders. This event, though, does not affect backorder lead-time because the new system schedules delivery times in a more accurate manner than the manual system. Experimenting with the BPISS model showed that a possible way of reducing backorders and consequently lead time is to increment the minimum stock level for each product. The results suggested a minimum stock level of 100 items for each product reduced the number of backorders having only $5 \%$ of backorders.

\section{Conclusions and further research}

This paper argues that despite the fact BP and IT interact in practice, existing $\mathrm{BP}$ and IS design 
approaches do not provide a clear guidance of which modeling techniques can be used to understand the complex relationships between them. Trying to address this problem, the ASSESS-IT framework proposed the use of BPS and CNS to coordinate the design of business process and IT simulation portraying in this way the effect that changes on any of these domains may have on the others.

The results derived from the ASSESS-IT framework suggest that the relationship between BP and IT can be described in some cases as the relationship between business processes and the information system that support those processes, and not as a three layered structured (BP, IS and CN) as it was initially thought in the ASSESS-IT framework. Furthermore, the results from the ASSESS-IT framework found that in order to depict the interactions between BP and IS it is necessary to portray IS nonfunctional requirements, in particular IS performance requirements. This paper used this knowledge to propose a new simulation framework, namely BPISS, to develop simulation models that depict business process and information systems performance.

The simulation results provided by the BPISS model shows that it is possible to obtain performance measurements of the IS and depict the way the insertion of IS affects BP performance. For example, the BPISS model provided quantifiable metrics of the IS, such as the number of backorders that the IS produces over a given period of time given a particular organizational context. These measurements helped to investigate the way IS may affect process performance. For example, new backordering delivery lead times that considered the effects that the IS has on the backordering process were obtained.

Another outcome of the BPISS framework is that it rendered evidence that suggests that simulation techniques can be used as an aid to design IS. For instance, the analysis of the traditional BPS model proposed in Tier Two of the BPISS framework provided more information to identify those areas where BP could be improved by the insertion of an IS. This information cannot be obtained by the used of traditional IS modeling techniques since they cannot always predict the way the IS will behave in practice. For example, based on the IS description provided by static models, it was thought that the IS would significantly reduce the number of backorders. After running the BPISS simulation model, it was shown that this was not the case.

The experiment showed that depicting the behavior of the IS and the effects that the latter would have on the processes is feasible, however, this was not and easy task. Despite the simplicity of the example used, the development of the model proved to be complex. It is thought that the higher the complexity of the IS the harder the construction of the simulation model. Hence, there is the need to test the BPISS framework with more complex IS. Regardless of these drawbacks, the results of the experiment showed that the new IS would improve the performance of the processes. Furthermore, It was noticed that the performance of the process did not depend only on the IS behavior but also on accurate changes to the business process themselves. This shows that business processes and the information systems are closely correlated and further research is needed to analyze this relationship in more detail. Finally, one of the major reasons of the complexity of this new approach was due to the fact that the discrete-event simulation tool used in our example was designed to model and simulate business process and offered limited capabilities to model and simulate information systems. Further research in this area is also needed in order to identify simulation tools that offer better capabilities to model the elements required to simulate IS or to propose the development of such tools.

\section{References}

[1] Davenport, T. H. and Stoddard, D. B. (1994) Reengineering: Business Change of Mythic Proportions? MIS Quarterly, 18(2), pp. 121-127.

[2] Davenport, T. H. (1993). Process Innovation: Reengineering Work through Information Technology. Boston, MA: Harvard Business School Press.

[3] Hammer, M. and Champy, J. (1993). Reengineering the Corporation: A Manifesto for Business Revolution. New York, NY: Harper Collins Publishers.

[4] Davenport, T. H. and Short, J. E. (1990) The New Industrial Engineering: Information Technology and Business Process Redesign. Sloan Management Review, 31(4), pp. 11-27.

[5] Childe, S. J., Bennett, J. and Maul, J. (1994) Frameworks for Understanding Business Process Re-engineering. International Journal of Operations \& Production Management, 14(12), pp. 22-34.

[6] Grover, V., Fielder, K. D. and Teng, J. T. C. (1994) Exploring the Success of Information Technology Enabled Business Process Reengineering. IEEE Transactions on Engineering Management, 41(3), pp. 276-284.

[7] Kettinger, W. J., Teng, J. T. C. and Guha, S. (1997) Business Process Change: A Study of Methodologies, Techniques, and Tools. MIS Quarterly, 21(1), pp. 55-80. 
[8] Painter, M. K., Fernades, R., Padmanaban, N. and Mayer, R. J. (1996) A Methodology for Integrating Business Process and Information Infrastructure Models. Charnes, J. M., Morrice, D. J., Brunner, D. T. and Swain, J. J. (Eds). Proceedings of the 1996 Winter Simulation Conference, Coronado, California, December 8-11. The Society for Computer Simulation International, pp. 1305-1312.

[9] Gladwin, B. and Tumay, K. (1994) Modelling Business Processes with Simulation Tools. Tew, J. D., Manivannan, S., Sadowski, D. A. and Seila, A. F. (Eds). Proceedings of the 1994 Winter Simulation Conference, Lake Buena Vista, FL, December 11-14. pp. 114-121.

[10] MacArthur, P. J., Crosslin, R. L. and Warren, J. R. (1994) A Strategy for Evaluating Alternative Information System Designs for Business Process Reengineering. International Journal of Information Management, 14(4), pp. 237-251.

[11] Warren, J. R., Crosslin, R. L. and MacArthur, P. J. (1995) Simulation Modeling for BPR: Steps to Effective Decision Support. Information Systems Management, 12(4), pp. 32-42.

[12] Hlupic, V. and Robinson, S. (1998) Business Process Modelling and Analysis Using DiscreteEvent Simulation. Medeiros, D. J., Watson, E. F., Carson, J. S. and Manivannan, M. S. (Eds). Proceedings of the 1998 Winter Simulation Conference, Washington, DC, December 13-16. The Society for Computer Simulation International, pp. 1363-1369.

[13] Giaglis, G. M. (1999) Dynamic Process Modelling for Business Engineering and Information Systems.Unpublished $\mathrm{PhD}$ Thesis, Brunel University, London.

[14] Law, A. M. and McComas, M. G. (1994) Simulation of Communication Networks: The State of the Art. IEEE Communications, 32, pp. 44-50.

[15] Banks, J., Carson, J. S., Nelson, B. L. and Nicol, D. M. (2000). Discrete-event System Simulation. 3rd ed. Upper Saddle River, NJ: Prentice-Hall.

[16] Serrano, A. (2002) Stochastic Information Technology Modelling for Business Processes.Unpublished PhD Thesis, Brunel University, London.

[17] Eatock, J., Paul, R. J. and Serrano, A. (2002) Developing a Theory to Explain the Insights Gained Concerning Information Systems and Business Processes Behaviour: The ASSESS-IT Project. Information Systems Frontiers, 4(3), pp. 303-316.

[18] Sommerville, I. (1997). Software Engineering. 5th ed. Wokingham: Addison-Wesley.
[19] Bennett, S., McRobb, S. and Farmer, R. (1999). Object-oriented Systems Analysis and Design Using UML. London: McGraw-Hill.

[20] Nuseibeh, B. and Easterbrook, S. (2000) Requirements Engineering. Proceedings of the 22th International Conference on Software Engineering, Limerick, Ireland, June 4-11. ACM Press, pp. 3546.

[21] Law, A. M. and Kelton, D. W. (2000). Simulation Modelling and Analysis. 3rd ed. New York, NY: McGraw-Hill. 